\title{
Comparison of Users' and Designers' Differences in Mobile Shopping App Interface Preferences and Designs*
}

\author{
$\mathrm{Yu} \mathrm{Fu}$ \\ Zhejiang University, Hangzhou, China \\ 21121054@zju.edu.cn
}

\author{
Dongliang Zhang \\ Zhejiang University, Hangzhou, China \\ dzhang@zju.edu.cn
}

\author{
Hao Jiang \\ Zhejiang University, Hangzhou, China \\ cuuacu@163.com
}

\begin{abstract}
Besides usability, visual appearance also plays an important role in influencing users' attitudes towards the mobile shopping apps. This article presents a pilot study that explores users' and designers' preferences for mobile shopping app user interfaces (UIs). The study consisted of two phases. (1) Eliciting participants' perception of UIs similarity by sorting, using DISTATIS and cluster analysis, the UIs similarity perceptual space was identified. (2) Eliciting participants' overall preference by rating. The results identified three typical UIs and the distribution of ideal preference UIs for users and designers. Last, users and designers' differences in the UI preference were discussed.
\end{abstract}

\section{KEYWORDS}

Mobile shopping, user interface(UI) design, perception difference

\section{INTRODUCTION}

Effective design of UI for mobile handheld devices facilitates user adoption of mobile commerce, and it is of great importance to leave a good impression on mobile shopping apps. When choosing how to attract the user's attention, designers face a variety of choices, and they generally make decisions based on anecdotes and intuition. Some design elements are widely used, perhaps because of designers believe they are effective at capturing users' attentions, but they end up annoying and repelling users. Consequently, this research aims to identify the differences, if any, between users' and designers' perceptions of mobile shopping app UIs. This study mainly focused on: (1) What are the types of shopping app UIs and what are the differences among these UIs design features? (2) Explore whether there are differences between users and designers' preferences for shopping app UIs.

\section{METHOD}

In the similarity experiment, the experimental element was the 40 most popular mobile shopping app UIs, on the Apple iTunes

This paper is published under the Creative Commons Attribution-NonCommercialNoDerivs 4.0 International (CC BY 4.0) license. Authors reserve their rights to disseminate the work on their personal and corporate Web sites with the appropriate attribution.

WWW'18 Companion April 23-27, 2018, Lyon, France.

(c) 2018 IW3C2 (International World Wide Web Conference Committee), published under Creative Commons CC BY 4.0 License.

ACM ISBN 978-1-4503-5640-4/18/04.

DOI: https://doi.org/10.1145/3184558.3186914.
Store of China. Participants included 24 designers and 24 users. Participants were asked to sort together shopping app UIs based on their perceived similarity.

In the preference experiment, 40 UIs were the same as the previous stage. Participants included 35 designers and 35 users. They were asked to use a 9-point Likert scale to rate the 40 shopping UIs according to their perceptual preferences.

\section{RESULTS AND DISCUSSIONS}

\subsection{Analysis of similarity in the UIs}

To explore participants' perceptions of the proximities among the shopping app UIs, the sorting data was analyzed using DISTATIS approach[1]. The results showed an UI similarity perceptual space from which critical dimensions influencing user perception of shopping UIs could be delineated. The factor scores are given in Table 1, and the projections of the UIs on the first three dimensions are shown in Fig. 1 and Fig. 2. Together, these three dimensions explained $78 \%$ of the inertia of the compromise matrix, which indicated that the interpretation of the results can be restricted to these three dimensions. Paired with the cluster analysis, the 40 UI samples plotted in this perceptual space were appropriately divided into three typical groups. The perceptual space from the DISTATIS analysis reveals distribution trends in the visual features of the UI representations. Cluster analysis showed three current typical shopping UIs.

\begin{tabular}{|c|c|c|c|}
\hline $\begin{array}{ll}\text { No. } & \text { of } \\
\text { Dimensions }\end{array}$ & 1 & 2 & 3 \\
\hline & 0.68 & 0.47 & 0.31 \\
\hline & $40 \%$ & $26 \%$ & $12 \%$ \\
\hline
\end{tabular}

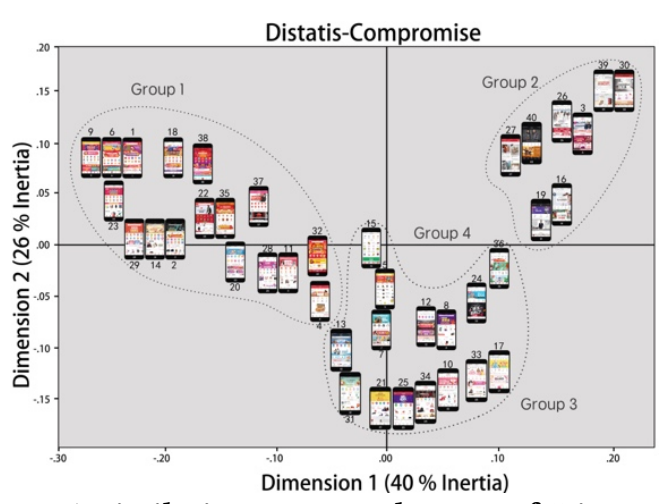

Figure 1: A similarity perceptual space of Dimensions 1 and 2 of the 40 UIs, obtained from DISTATIS. 


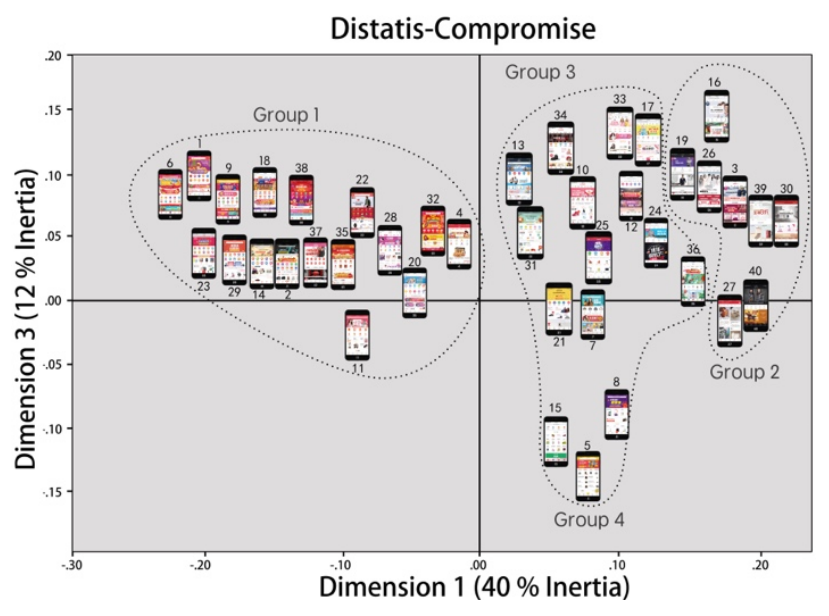

Figure 2: A similarity perceptual space of Dimensions 1 and 3 of the 40 UIs, obtained from DISTATIS.

\subsection{Preference mapping analysis of the UIs}

User participants preferred UI No. 22 the most $(M=5.94 ; S D=1.84)$ while designer participants liked UI No. 16 the most $(M=5.83$; $\mathrm{SD}=2.36$ ). In order to more detailed understanding of the differences between users' and designers' preferences and design features of ideal shopping UIs, the three dimensions of perceptual space were needed. Therefore, according to user and designer participants' preferences score for 40 interface samples, we obtain a ternary diagram[2] (Fig. 3), which shows the participants' preferences toward the 40 UIs. The ternary diagram is a triangular diagram which displays the proportion of three variables that sum to a constant and which does so using barycentric coordinates[3]. In Fig. 3, the three variables represent the three preference dimensions (Like, So-So, Dislike), each of these points is the composition of three variable. Based on the similarity space previously plotted by DISTATIS, three UI groups were presented in different colors and different symbols in Fig. 3. It shows the users' preferences map is relatively dispersed, while the designers' preferences map is relatively concentrated. Based on the three UI types, it is obvious that users preferred the UIs in group 1, while designers preferred the UIs that are relatively focus on group 2 . It indicates that users and designers have significant disagreements about their preferences towards to the UIs in group 1 and group 2. The common characteristics of users preferred UIs included the use of warm colors, high density and rich functions (search bar $+1 / 5$ banner+push bar +2 rows of icon + push module + bottom navigation). However, the common features of designers preferred interfaces included the use of neutral colors, low density and simple function (top navigation, +2 big banners + bottom navigation).

\section{CONCLUSIONS}

This paper describes a case study exploring the differences between users' and designers' perceptions of shopping app UI designs. Shopping app UIs, including various design features, were chosen as elements. Participants were asked to sort these
UIs according to visual similarities and differences. Then three typical shopping UIs were identified through cluster analysis. Next, users' and designers' preferences of the UIs were identified. Users mostly preferred interfaces which mainly focus on group 1, while designers' preference of interfaces were relatively concentrated in group 2. Designers tended to prefer the UI with overall layout, while users preferred the UI with subdivision layout. The results highlight the perception differences between users and designers in shopping app UI preferences, which can help designers better understand the target users and reduce the perception differences with users.
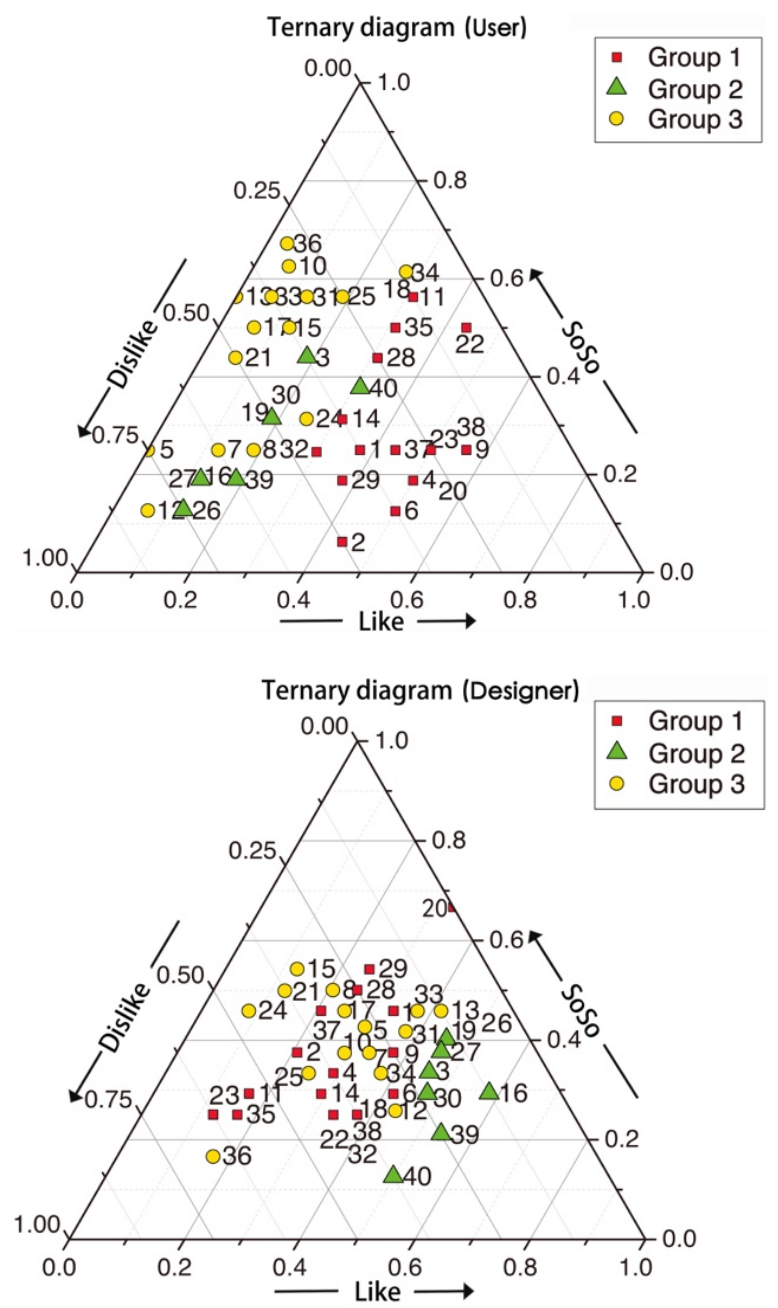

Figure 3: User and designer participants' preferences for 40 interfaces.

\section{REFERENCES}

[1] Abdi, H., et al. 2007. Analyzing assessors and products in sorting tasks: DISTATIS, theory and applications. Food quality and preference, 18(4): p. 627-640.

[2] West, D.2012:Ternary equilibrium diagrams. Springer Science \& Business Media.

[3] Weisstein, E.W. 2014.Ternary Diagram. From MathWorld,A Wolfram Web Resource: http://mathworld.wolfram.com/TernaryDiagram.html. 